\title{
Segmento ST vemos, coronarias no sabemos. Reporte de caso de infarto agudo de miocardio con arterias coronarias no obstruidas
}

\author{
ST segment we see, coronary we don't know. Case report of acute myocardial infarction \\ with non-obstructed coronary arteries
}

Carlos Haro-Haro ${ }^{1 *}$, María del R. Ramírez-Galindo y Anuar Delahanty-Delgado²

${ }^{1}$ Servicio de Medicina de Urgencias; ${ }^{2}$ Servicio de Cardiología. Hospital General 450, Durango, México

\section{Introducción}

El diagnóstico de síndrome coronario agudo se establece por evidencia de lesión miocárdica aguda acompañada de datos clínicos de isquemia miocárdica, nuevos cambios en el electrocardiograma (ECG), pérdida de miocardio viable presente en las imágenes 0 identificación de trombos coronarios ${ }^{1}$, y se define como daño miocárdico la detección de un valor de troponina cardiaca (cTn) por encima del percentil $99^{2}$. El 90\% de los pacientes con infarto agudo de miocardio (IAM) sometidos a coronariografía tienen una obstrucción evidente de arterias coronarias (estenosis > 50\%); en el $10 \%$ restante el grado de estenosis es $<50 \%$ y se denomina IAM con arterias coronarias no obstructivas (MINOCA, myocardial infarction with non-obstructive coronary arteries), una afección clínica desconcertante, con una prevalencia entre el $2 \%$ y el $10 \%{ }^{3}$, de predominio en jóvenes, mujeres y con menos factores de riesgo cardiovascular ${ }^{1}$.

\section{Presentación del caso}

Varón de 30 años, previamente sano, niega consumo de drogas, tabaquismo activo. Acude al servicio de urgencias por un cuadro clínico de 60 minutos de evolución, caracterizado por dolor precordial, de tipo opresivo, retroesternal, de intensidad 10/10 en la escala visual analógica (EVA), irradiado a la extremidad superior izquierda, acompañado de criodiaforesis, náuseas y palidez generalizada. Signos vitales al ingreso: presión arterial 111/69 mmHg, frecuencia cardiaca 73 latidos por minuto, frecuencia respiratoria 21 respiraciones por minuto, temperatura $36.7^{\circ} \mathrm{C}$, Glasgow $15 / 15$ y saturación de oxígeno $96 \%$ sin oxígeno suplementario. Se realiza ECG de 12 derivaciones, el cual muestra elevación del segmento ST en cara inferior (Fig. 1). Los laboratorios de ingreso reportan leucocitos $11 \times 10^{3}$, neutrófilos $82.1 \%$, linfocitos $10.1 \%$, hemoglobina $15.6 \mathrm{~g} / \mathrm{dl}$, hematocrito $46.4 \%$, plaquetas 166,000, proteína C reactiva $18 \mathrm{mg} / \mathrm{l}$, creatinina $0.8 \mathrm{mg} /$ $\mathrm{dl}$, urea $23.5 \mathrm{mg} / \mathrm{dl}$ y nitrógeno ureico en sangre $11 \mathrm{mg} /$ dl. Se realiza una radiografía de tórax, sin alteraciones.

\section{Abordaje}

Por los cambios del ECG inicial, el cuadro clínico de dolor precordial de características isquémicas, el tiempo de evolución de 60 minutos y el reporte del perfil cardiológico con cTnl $5.23 \mathrm{ng} / \mathrm{dl}$, creatina fosfocinasa MB $37 \mathrm{U} / \mathrm{l}$ y péptido natriurético cerebral $88.9 \mathrm{pg} / \mathrm{ml}$, 


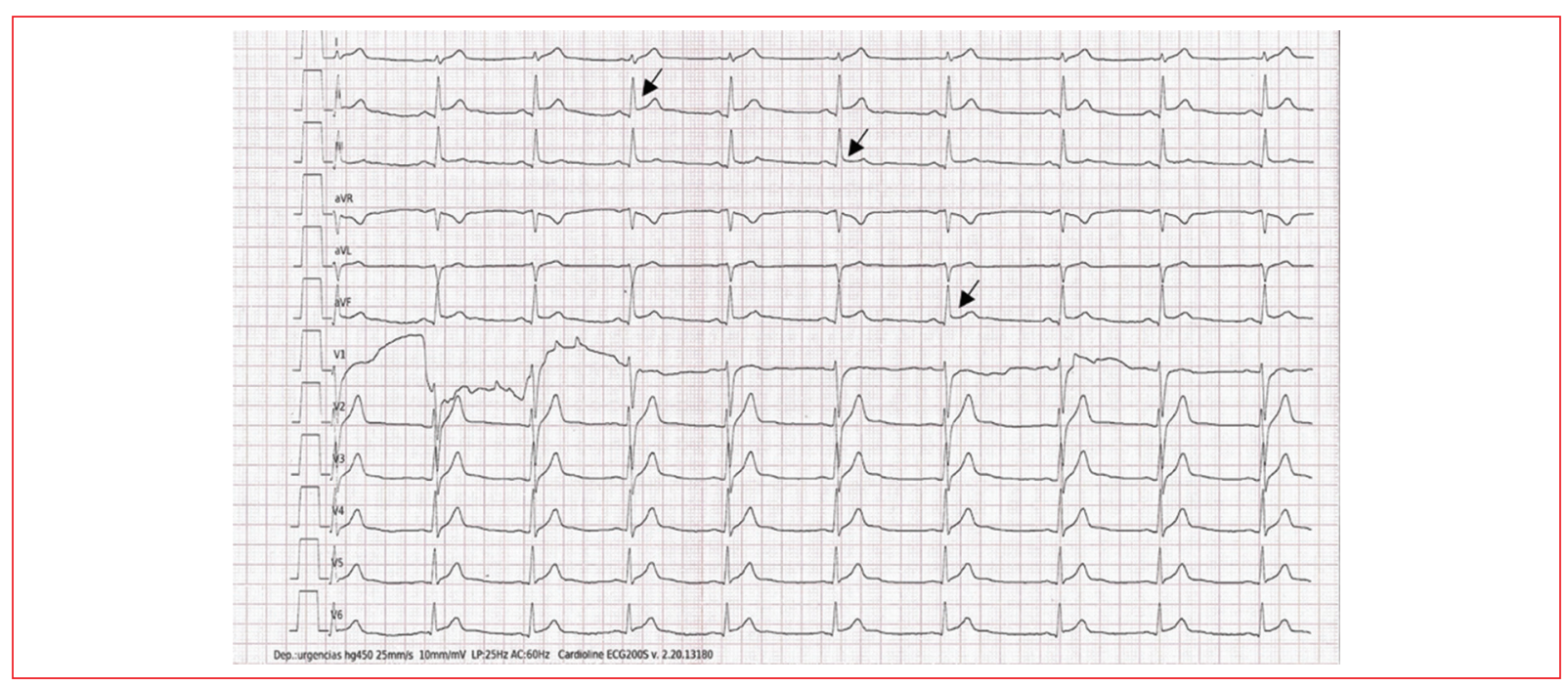

Figura 1. ECG de ingreso a Urgencias. Elevación del punto J $0.2 \mathrm{mV}$ en cara inferior (flechas negras).

se integra diagnóstico de IAM con elevación del segmento ST de cara inferior. Al estar dentro de la ventana trombolítica, se decide trombólisis intravenosa con tenecteplasa.

\section{Diagnósticos diferenciales}

Se debe realizar con las causas de dolor torácico de origen cardiaco y no cardiaco que ponen en peligro la vida, como angina de pecho, tromboembolia pulmonar, síndrome aórtico, pericarditis, miocarditis y úlcera perforada, entre otras. Algunas de estas patologías cursan también con elevación de las troponinas sin enfermedad coronaria.

\section{Tratamiento}

Se inició manejo con ácido acetilsalicílico, clopidogrel, enoxaparina y atorvastatina, y se realizó trombólisis intravenosa con tenecteplasa (40 mg), a los $94 \mathrm{mi}-$ nutos de su ingreso y 154 minutos del inicio del dolor. Se realizaron ECG de control a 30,60 y 90 minutos (Fig. 2). Presentó descenso del segmento ST del $50 \%$, disminución del dolor precordial a intensidad $4 / 10$ y cese del cortejo neurovegetativo a los 90 minutos. Se reportó Glasgow 15/15, presión arterial 101/65 mmHg, frecuencia cardiaca 70 latidos por minuto, frecuencia respiratoria 16 respiraciones por minuto, temperatura $36.7{ }^{\circ} \mathrm{C}$, saturación de oxígeno del $95 \%$ sin oxígeno suplementario, glucemia capilar $117 \mathrm{mg} / \mathrm{dl}$ y gingivorragia mínima.

\section{Desenlace y seguimiento}

Como parte del protocolo diagnostico se realizó coronariografía 5 horas posteriores a la trombólisis, la cual reportó tronco coronario izquierdo bifurcado sin lesiones, arteria coronaria descendente anterior sin lesiones significativas, arteria circunfleja dominada sin lesiones, arteria coronaria derecha dominante sin lesiones significativas y sin presencia de trombo (Fig. 3). El ecocardiograma transtorácico se describe con ventrículo izquierdo no dilatado, movilidad global y segmentaria conservada, fracción de eyección del ventrículo izquierdo $55 \%$, patrón de llenado normal, válvulas aórtica, tricúspide, mitral y pulmonar normales, pericardio normal. Transcurridas 24 horas presenta un nuevo episodio de dolor precordial de intensidad 7/10 en la EVA, acompañado de diaforesis, con duración de 10 minutos, que mejora con la administración de nitratos. El ECG presenta elevación del segmento ST en cara inferior $0.3 \mathrm{mV}$, y la cTnl se reporta en $11.61 \mathrm{ng} / \mathrm{ml}$ y posteriormente, a las 48 horas, en $15.86 \mathrm{ng} / \mathrm{ml}$. Se establece diagnóstico de IAM con arterias coronarias no obstruidas de tipo vasoespasmo coronario y se inicia manejo con antagonista del calcio no dihidropiridínico, con buena respuesta. Al cuarto día de estancia hospitalaria presenta evolución favorable, sin más episodios de dolor o equivalentes anginosos, por lo que se egresa con seguimiento por cardiología. 


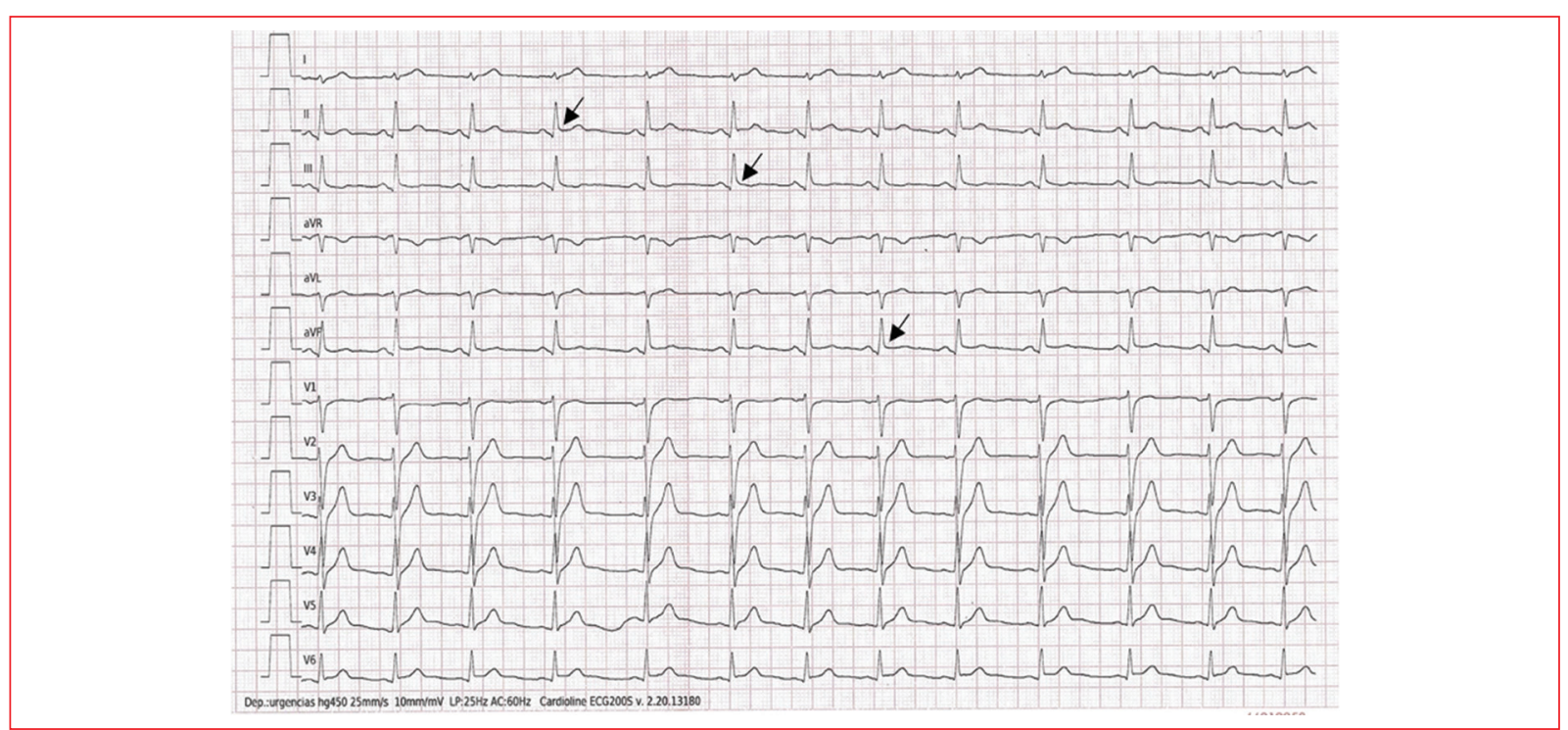

Figura 2. ECG 90 minutos post trombolísis. Descenso del punto $\mathrm{J}$ a $0.1 \mathrm{mV}$ en cara inferior (flechas negras).

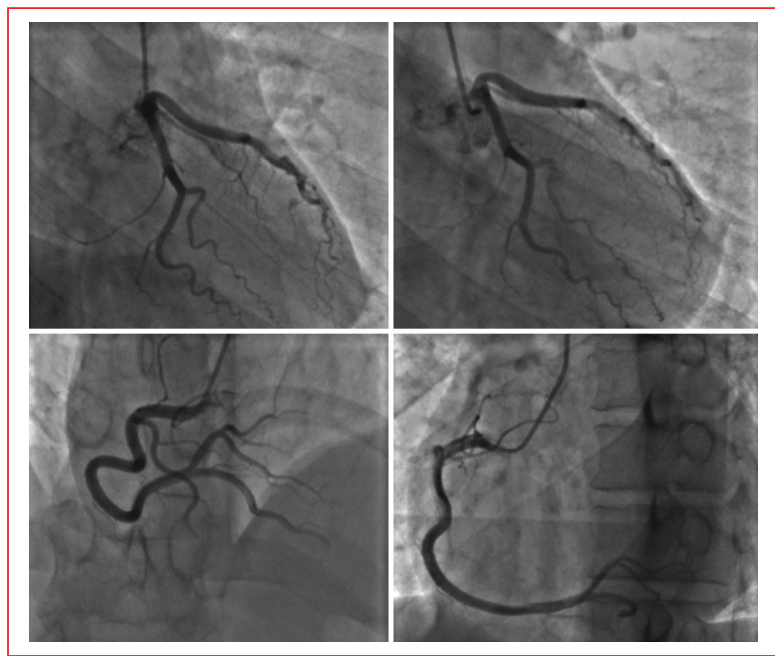

Figura 3. Coronariografía 5 horas post trombolísis. Arterias coronarias sin lesiones, no se observan defectos de llenado ni obstrucción del flujo del medio de contraste.

\section{Discusión}

El término MINOCA (infarto de miocardio sin arterias coronarias obstructivas) fue utilizado por primera vez en 2013 por Beltrame ${ }^{3}$. Actualmente se define MINOCA como la documentación clínica de IAM junto con la demostración de arterias coronarias sin lesiones obstructivas significativas ${ }^{4}$. El MINOCA corresponde a un infarto de tipo 2 de la actual definición universal de infarto, con un desequilibrio entre la oferta y la demanda miocárdica de oxígeno ${ }^{5}$.

Se conocen dos mecanismos fisiopatológicos del MINOCA que se traducen en dos patrones de lesión: 1) el patrón epicárdico, cuyas causas son disrupción de la placa, disección coronaria, vasoespasmo coronario y embolia coronaria, y 2) el patrón microvascular, cuyas causas involucran espasmo microvascular y embolia coronaria de pequeños vasos de múltiples etiologías ${ }^{6}$.

El vasoespasmo coronario es la causa más frecuente de MINOCA de patrón epicárdico, es más común en hombres que en mujeres, de entre 40 y 70 años, y predomina en japoneses $(24,3 \%)$, seguidos de taiwaneses $\left(19,3 \%\right.$ y caucásicos $(7,5 \%)^{5,6}$. El estudio VIRGO reportó que las mujeres y los pacientes no blancos tenían mayores posibilidades de presentar MINOCA'. La incidencia aumenta ligeramente en verano y otoño, siendo más frecuente por la mañana ${ }^{7}$.

El diagnóstico de MINOCA se basa en la evidencia de IAM (cambios en el ECG y biomarcador cardiaco anormal) y se establece de forma definitiva en la coronariografía al observar arterias no obstruidas (estenosis $<50 \%)^{3}$.

En el ECG se buscan los signos de isquemia miocárdica, aunque es frecuente encontrar cambios difusos del ST que no respetan territorios vasculares (orientativo de miopericarditis) y presencia de arritmias con potencial embolígeno ${ }^{4}$. 
El MINOCA puede presentarse con o sin elevación del segmento ST independientemente de la etiología. La probabilidad de encontrar enfermedad arterial coronaria no obstructiva en mujeres es similar tanto para IAM sin elevación del ST como para IAM con elevación del ST, mientras que en los hombres es más frecuente en IAM con elevación del ST'.

Además de la troponina, son útiles para un diagnóstico más específico el péptido natriurético cerebral, el dímero $\mathrm{D}$, la proteína $\mathrm{V}$ Leiden, la proteína $\mathrm{C}$, la proteína $\mathrm{S}$, el factor lúpico, los anticuerpos anticardiolipina y los hemocultivos en caso de fiebre ${ }^{4}$.

El ecocardiograma ayuda a determinar y detectar trombos intraventriculares, masas cardiacas (mixoma, fibroelastoma papilar, etc.), vegetaciones, alteraciones valvulares, placas en la aorta ascendente y shunts derecha-izquierda que pueden causar embolias coronarias ${ }^{4,7}$.

La angiografía coronaria define al MINOCA por la ausencia de enfermedad arterial coronaria obstructiva (estenosis $\leq 50 \%$ ), en cualquier arteria potencialmente relacionada con un infarto; esto incluye arterias coronarias normales (estenosis $\leq 30 \%$ ) o ateromatosis coronaria leve (estenosis $>30 \%$ y $<50 \%$ ). En la angiografía, la causa de un diagnóstico específico no es evidente y existe la necesidad de evaluar al paciente con pruebas complementarias ${ }^{7}$; no se recomienda la prueba de la acetilcolina para la detección de vasoespasmo en la fase aguda del IAM ni en pacientes inestables ${ }^{4,8}$.

El diagnóstico de angina vasoespástica se basa en que los episodios espontáneos de angina en reposo se asocian con cambios en el segmento ST y en su respuesta a los nitratos de acción corta ${ }^{8}$.

La resonancia magnética cardiaca, la tomografía de coherencia óptica (OCT), el ultrasonido intravascular (IVUS) y la ventriculografía izquierda son herramientas de diagnóstico en la unidad de cuidados coronarios ${ }^{5}$. La resonancia magnética cardiaca permite la aproximación diagnóstica en el $70 \%$ de los casos ${ }^{4}$; el realce tardío de gadolinio en el subendocardio sugiere una causa isquémica de lesión, aunque no identifica la causa particular de la isquemia, mientras que una localización subepicárdica orienta hacia una miocardiopatía ${ }^{8}$.

El manejo terapéutico del MINOCA exige el conocimiento de la causa fisiopatológica, pero los dos pilares son el tratamiento antiaterosclerótico y el tratamiento antiisquémico ${ }^{4}$. El uso de estatinas, inhibidores de la enzima convertidora de angiotensina y bloqueadores de los receptores de la angiotensina II tiene beneficios significativos para mejorar el pronóstico a largo plazo de los pacientes con MINOCA, mientras que el ácido acetilsalicílico, el clopidogrel y los betabloqueadores no han mostrado ninguna mejora en el pronóstico ${ }^{9}$.

Los pacientes con ángor y elevación de la troponina son tratados con terapia antiplaquetaria simple o doble desde el ingreso (ácido acetilsalicílico y clopidogrel o ticagrelor) y anticoagulados con heparina ${ }^{4}, y$ después de la coronariografía pueden considerarse dos posibilidades:

- Si se realizó IVUS/OCT y se detectaron placas accidentadas, se recomienda mantener la doble terapia antiagregante durante 1 año.

- Si no se realizó IVUS/OCT, evaluar diagnósticos diferenciales, y si no puede descartarse un evento de placa, se recomienda continuar con doble antiagregación durante 1 año.

En los pacientes con vasoespasmo coronario se recomiendan los nitratos y los bloqueantes del calcio no dihidropiridínicos, como el verapamilo o el diltiazem, $270 \mathrm{mg} / \mathrm{día}$, y si no hay respuesta o persisten los síntomas se puede aumentar a $540 \mathrm{mg} / \mathrm{día}$; en caso de no haber mejoría podrían agregarse amlodipino o cilostazol, o ambos ${ }^{4,9}$.

\section{Perspectivas del paciente}

Los primeros 3 meses después del vasoespasmo coronario son el período crítico con mayor riesgo de eventos cardiovasculares. El abandono del hábito tabáquico y el tratamiento con bloqueantes del calcio no dihidropiridínicos son los factores pronósticos más determinantes. La muerte cardiaca ocurre en el $0-10 \%$ de los casos, y la angina vasoespástica recurrente aparece en el 3.9$18.6 \%^{6}$. Los pacientes con MINOCA tienen buenos resultados, incluida baja mortalidad (3.2-4.5\%), en comparación con aquellos con infarto de miocardio y enfermedad arterial coronaria obstructiva, así como adecuado estado psicosocial y funcional ${ }^{2}$. El perfil clínico y el tratamiento de los pacientes es variable, y es necesaria una caracterización más específica de esta patología; la alta sospecha por parte del personal médico es lo que determina la conducta de estudio de estos pacientes.

\section{Financiamiento}

Los recursos de este estudio fueron proporcionados por los autores.

\section{Conflicto de intereses}

No se reportaron conflictos de intereses durante la realización de este trabajo. 


\section{Responsabilidades éticas}

Protección de personas y animales. Los autores declaran que para esta investigación no se han realizado experimentos en seres humanos ni en animales.

Confidencialidad de los datos. Los autores declaran que en este artículo no aparecen datos de pacientes.

Derecho a la privacidad y consentimiento informado. Los autores declaran que en este artículo no aparecen datos de pacientes.

\section{Bibliografía}

1. Vidal-Pérez R, Casas CAJ, Agra-Bermejo RM, Álvarez-Álvarez B, Grapsa J, Fontes-Carvalho R, et al. Myocardial infarction with non-obstructive coronary arteries: a comprehensive review and future research directions. World J Cardiol. 2019;11:305-15.
2. Safdar B, Spatz ES, Dreyer RP, Beltrame JF, Lichtman JH, Spertus JA, et al. Presentation, clinical profile, and prognosis of young patients with myocardial infarction with nonobstructive coronary arteries (MINOCA): results from the VIRGO study. J Am Heart Assoc. 2018;7:e009174.

3. Beltrame JF. Assessing patients with myocardial infarction and nonobstructed coronary arteries (MINOCA). J Intern Med. 2013;273:182-5.

4. Cohen Arazi $H$, Iglesias R, Duronto E, Lescano A, Campisi R, Deviggiano A et al.; GREECO (Grupo de Estudio de Enfermedades Coronarias). Isquemia miocárdica sin lesiones coronarias obstructivas: MINOCA-INOCA. Revisión para la toma de decisiones. Medicina (B Aires). 2020;80:253-70.

5. Thygesen K, Alpert JS, Jaffe AS, Chaitman BR, Bax JJ, Morrow DA, et al. Fourth Universal Definition of Myocardial Infarction (2018). J Am Coll Cardiol. 2018;72:2231-64.

6. Ache Y, Guamán C, Viñole L, Vignolo G. Infarto agudo de miocardio sin lesiones coronarias obstructivas - MINOCA: un enigma para el cardiólogo clínico. Rev Urug Cardiol. 2020;3(1). Epub 01-Abr-2020. http://dx. doi.org/10.29277/cardio.35.1.11.

7. Agewall S, Beltrame JF, Reynolds HR, Niessner A, Rosano G, Caforio ALP et al. ESC working group position paper on myocardial infarction with non-obstructive coronary arteries. Eur Heart J. 2017;38:143-53.

8. Matta A, Bouisset F, Lhermusier T, Campelo-Parada F, Elbaz M Carrié D, et al. Coronary artery spasm: new insights. J Interv Cardiol. 2020;2020:5894586.

9. Abdu FA, Mohammed AQ, Liu L, Xu Y, Che W. Myocardial infarction with nonobstructive coronary arteries (MINOCA): a review of the current position. Cardiology. 2020;145:543-52. 